\title{
Crescentic IgA nephropathy associated with pleural tuberculosis
}

\author{
Marta R Marques ${ }^{1}$, Pedro R Pereira ${ }^{2}$, Sofia Marques $^{3}$ \\ ${ }^{1}$ Department of Internal Medicine, Centro Hospitalar Póvoa de Varzim/Vila do Conde. Póvoa de Varzim. Portugal. \\ 2 Department of Pathology, Centro Hospitalar São João. Porto. Portugal. \\ ${ }^{3}$ Department of Nephrology, Centro Hospitalar S. João. Porto. Portugal.
}

\section{ABSTRACT}

Crescentic IgA nephropathy represents an aggressive glomerular injury which can cause renal failure unless diagnosed and treated rapidly. There is currently no consensus on the most effective treatment strategy, although immunosuppressive drugs are usually used. Tuberculosis is rarely associated with glomerulonephritis and raises difficult questions about the safety and usefulness of immunosuppressive agents. We report a rare case of rapidly progressive renal failure associated with pleural tuberculosis in a 68-year-old man. The identification of crescents and fibrinoid necrosis in 12 out of 21 glomeruli and IgA deposits in the renal biopsy suggested the diagnosis of crescentic IgA nephropathy. Corticosteroids in combination with antituberculosis drugs were started with significant improvement in his general condition and cure of his pleural tuberculosis but without recovery of kidney function.

Keywords: Crescentic glomerulonephritis, IgA nephropathy; immunosuppression; renal failure; pleural tuberculosis.

\section{INTRODUCTION}

Immunoglobulin A nephropathy (IgAN), the most common primary glomerulopathy worldwide, is an autoimmune disease whose pathogenesis is described as a "multi-hit" process resulting in IgA glomerular deposition ${ }^{1}$. IgAN is usually a primary disease but may be associated with different nonrenal diseases (secondary IgAN), such as chronic liver diseases, cancer and infections ${ }^{2}$. To provide the best treatment, it is very important to clarify its pathogenesis.

A wide range of histological changes have been described: from near normal to severe proliferative glomerulonephritis with cellular crescents. This translates into different clinical courses (from asymptomatic microscopic hematuria to rapidly progressive glomerulonephritis or nephrotic syndrome) and variable prognosis: most forms are benign without significant kidney injury, whereas others lead to significant kidney injury and progression to end-stage renal disease ${ }^{3}$.

Crescentic IgAN (C-IgAN), usually defined as the presence of crescents in over $50 \%$ of the glomeruli, is rare and often presents as rapidly progressive kidney failure ${ }^{4}$. C-IgAN may likely benefit from combined imunossupression ${ }^{5}$.

We report a case of active tuberculosis and acute kidney injury (AKI) whose biopsy revealed a C- IgAN and discuss a possible association between the two diseases and treatment options.

\section{CASE REPORT}

A 68-year-old man with arterial hypertension, dyslipidemia and ischemic heart disease was submitted to coronary artery bypass surgery. It complicated with Dressler's syndrome and he started twice-daily $600 \mathrm{mg}$ ibuprofen. Until then his serum creatinine was normal $(0.9 \mathrm{mg} / \mathrm{dL})$ and no urine analysis was available. Two months later, still on ibuprofen, he was re-admitted to the hospital for AKI [serum creatinine $(\mathrm{s} C \mathrm{C}) 3.1 \mathrm{mg} / \mathrm{dl}$ ], hematuria and nephrotic-range proteinuria (proteinuria $10 \mathrm{~g} / 24 \mathrm{~h}$ ). The laboratory evaluation for autoimmune diseases was negative and these changes were thought to be due to a hypersensitivity reaction to ibuprofen but no kidney biopsy was performed at the time. He empirically started high-dose corticosteroids which were very slowly reduced over the next months with rapid partial improvement of the kidney function ( $\mathrm{s} C \mathrm{r}$ decreased and remained at $1.6 \mathrm{mg} / \mathrm{dL}$ ) and urinary changes.

Eight months later, he presented to the emergency department with fever and macroscopic hematuria. At that time he was still taking $10 \mathrm{mg}$ of prednisolone. On physical examination, he was febrile (38.6ㅇ) and breath sounds were decreased at the left lung base.

Laboratory investigation showed leukocytosis $\left(15000\right.$ cells $\left./ \mathrm{mm}^{3}\right)$, normal C-reactive protein, hypoalbuminemia (25.2g/L), AKI $(\mathrm{sCr}$ $3.2 \mathrm{mg} / \mathrm{dl})$, hematuria, leukocyturia and proteinuria $(4 \mathrm{~g} / \mathrm{L})$. Serum electrolytes were within normal limits.

The chest X-ray revealed a pleural effusion in the lower half of the left lung which by thoracic CT scan suggested empyema. Additionally, there were scattered bilateral centrilobular micronodules (Figure 1). Multiple cultures of sputum, bronchoalveolar lavage and blood were negative for both bacteria and mycobacteria. Pleural fluid analysis was consistent with a lymphocytic exsudate with normal $\mathrm{pH}$, low glucose $(48 \mathrm{mg} / \mathrm{dl}$ ) and an increased adenosine deaminase level (102 $\mathrm{UI} / \mathrm{I})$. No acid-fast bacilli were observed and polymerase chain reaction for Mycobacterium tuberculosis (MTB) DNA was negative. Histological examination of the pleural biopsy showed mixed inflammatory infiltrate without caseating granulomas. 


\section{Figure 1}

Thoracic CT scan reveals a clear left pleural effusion with features suggesting empyema

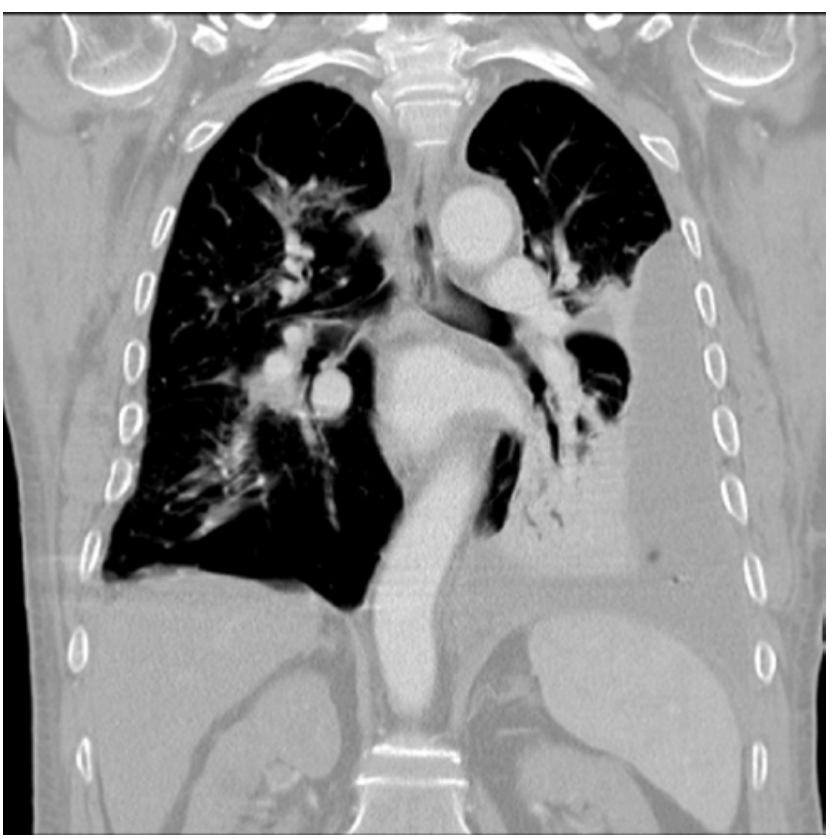

Renal ultrasonography showed normal-sized kidneys with normal parenchymal thickness but increased echogenicity. Markers of immune-mediated glomerulonephritis (antinuclear, antineutrophil cytoplasmic, antiglomerular basement membrane and anti-streptolysin 0 antibodies as well as anti-dsDNA, anti-ENA, rheumatoid factor and anti-CCP) were all negative. Serum complement levels, immunoglobulins and the serum protein electrophoresis were normal.
Serological tests were negative for syphilis, hepatitis B, C and human immunodeficiency virus.

At hospital admission, amoxicillin/clavulanate and clindamycin were started. Due to persistent pleural lymphocytic exsudate, antituberculosis treatment (ATT) was empirically started, cultures were repeated, and one did come out positive for MTB, confirming pleural tuberculosis.

During the first 2 weeks, renal function worsened, requiring the start of hemodialysis. A renal biopsy was performed and revealed crescent formation and fibrinoid necrosis in 12 out of 21 glomeruli. Immunofluorescence identified IgA (++) and C $3 \mathrm{C}(++)$ mesangial deposits. Electronic microscopy identified mesangial and scarce subendothelial, subepithelial and intramembranous deposits. The interstitium showed no significant inflammatory infiltrate or granulomas (Fig.2). The diagnosis of C-IgAN was established, and high-dose corticosteroids were initiated (intravenous pulses followed by $1 \mathrm{mg} / \mathrm{kg} /$ day oral prednisolone). Upon starting ATT and corticosteroids, the patient's clinical condition improved, and 6 months later pleural tuberculosis was considered cured. However, he did not recover kidney function and remained on hemodialysis.

\section{DISCUSSION}

IgAN is an important cause of chronic kidney disease and end-stage kidney failure that results from glomerular deposition of IgA-containing nephritogenic immune complexes. ${ }^{1}$

The Oxford Classification ${ }^{6}$, a grading system for histologic findings in primary IgAN, originally included mesangial hypercellularity, segmental sclerosis, interstitial fibrosis/tubular atrophy and endocapillary proliferation but did not account for glomerular crescents. However, several authors ${ }^{7}$ have shown that the presence of cellular or fibrocellular crescents negatively affects renal outcomes independently of

\section{Figure 2}

Kidney biopsy histology of crescentic glomerulonephritis (a) showing five glomeruli, three of them with cellular crescents (Masson trichrome, $x 40$ ), and at a closer look (b, x100 and c, x 200) diffuse endo and extracapillary proliferation with areas of fibrinoid necrosis (H.E., x100 and $x 200$ )
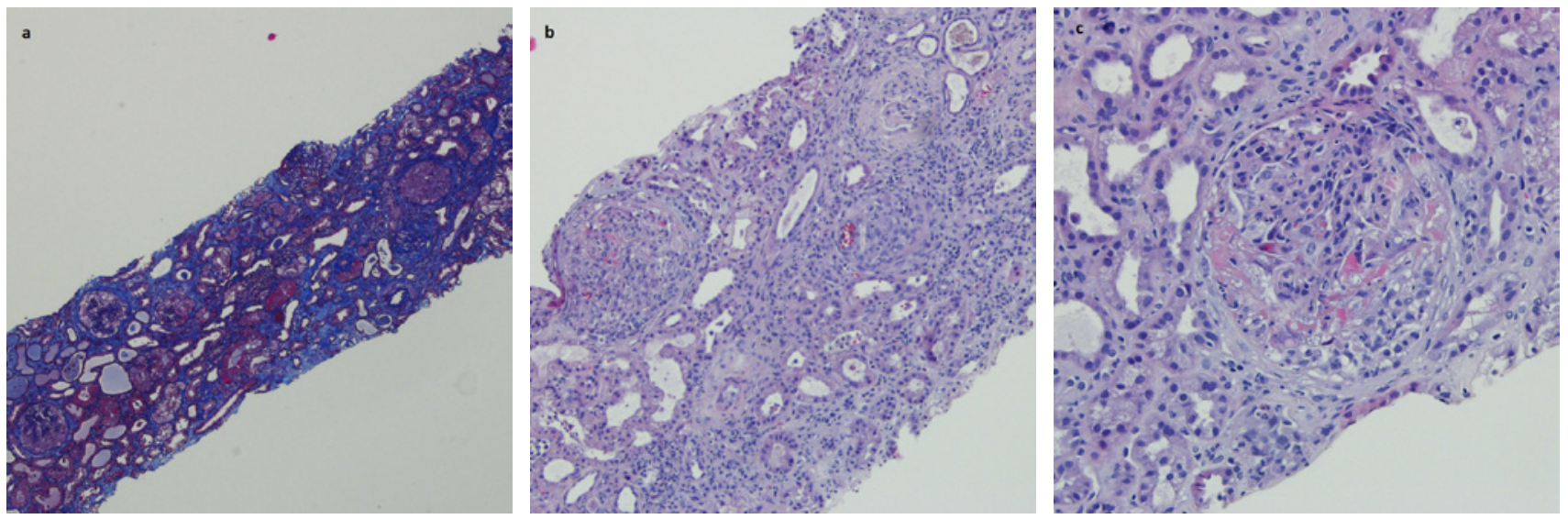


\section{Figure 3}

Electron micrograph of glomerular tissue showing wrinkling of the glomerular basement membrane, subendothelial and mesangial deposits

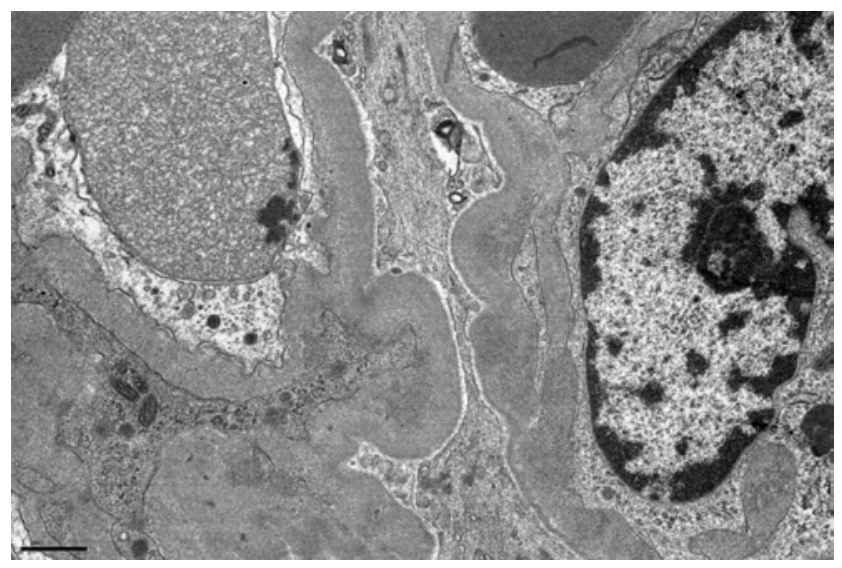

clinical or other histological parameters and so crescents were recently added to the original score ${ }^{8}$.

Its treatment includes renin-angiotensin system blockers, corticosteroids for patients with poor prognostic factors ${ }^{5}$ and less proven strategies such as omega- 3 fatty acids ${ }^{9}$. In patients with moderate proteinuria and chronic kidney disease stages 1 through 3, the addition of corticosteroids to optimized supportive care does not seem to carry benefits ${ }^{10}$.

Crescentic glomerulonephritis is a life-threatening condition with a poor outcome if treatment is delayed and the patient develops renal failure. For C-IgAN, cyclophosphamide combined with corticosteroids may reduce proliferative lesions, proteinuria and stabilize renal function $^{11}$. Secondary C-IgAN represents a greater challenge especially when triggered by an infection.

Some cases of IgAN have been reported in the setting of tuberculosis which may be initiated by the humoral immune response against MTB and the production of IgA immune complexes ${ }^{12}$. Rapidly progressive glomerulonephritis caused by MTB is extremely rare and most cases represent acute postinfectious glomerulonephritis (APIGN) ${ }^{13,14}$. IgA-dominant APIGN is most common in old diabetic patients with staphylococcal infections. It presents with hypocomplementemia, diffuse endocapillary proliferation by light microscopy, strong staining for C3 and IgA by immunofluorescence and hump-shaped subepithelial deposits seen with electron microscopy ${ }^{15}$. Our case lacks some of these usual features. Conversely, the previous episode of AKI and remaining chronic kidney disease and hematoproteinuria suggested a previous IgAN which was triggered or acquired an aggressive behaviour in the presence of tuberculosis. An additional case describing C-IgAN associated with tuberculosis was found in the literature ${ }^{14}$.

ATT was empirically started before immunosuppression due to the strong clinical suspicion of tuberculosis. Corticosteroids were soon increased after the diagnosis of C-IgAN but other immunosuppressants such as cyclophosphamide were considered unsafe in the context of active tuberculosis. Despite corticosteroids and ATT, the patient did not recover from AKI and maintenance hemodialysis was instituted.

Tuberculosis-related C-IgAN is definitely a double diagnostic challenge which raises difficult questions about the safety and usefulness of immunosuppression.

\section{Disclosure of potential conflicts of interest: none declared.}

\section{References}

1. Magistroni R, D'Agati VD, Appel GB, Kiryluk K. New developments in the genetics, pathogenesis, and therapy of IgA nephropathy. Kidney Int. 2015;88:974-989.

2. Saha MK, Julian BA, Novak J, Rizk DV. Secondary IgA nephropathy. Kidney Int. 2018;94(4):674-681.

3. Rodrigues JC, Haas M, Reich HN. IgA Nephropathy. Clin J Am Soc Nephrol. 2017;12(4):677-686.

4. Silva GE, Costa RS, Lobato ET, et al. A case series of diffuse crescentic IgA nephropathy: an omitted entity in the Oxford classification. Int J Clin Exp Pathol. 2016;9(2):1909-1916.

5. Kidney Disease: Improving Global Outcomes (KDIGO) Glomerulonephritis Work Group. KDIGO Clinical Practice Guideline for Glomerulonephritis. Kidney inter, Suppl. 2012;2:139-274.

6. Roberts IS, Cook HT, Troyanov S, et al. The Oxford classification of IgA nephropathy: Pathology definitions, correlations, and reproducibility. Kidney Int. 2009;76(5):546-556.

7. Haas M, Verhave JC, Liu ZH, Alpers CE, et al. A Multicenter Study of the Predictive Value of Crescents in IgA Nephropathy. J Am Soc Nephrol. 2017;28(2):691-701.

8. Trimarchi H, Barratt J, Cattran DC, et al. Oxford classification of IgA nephropathy 2016: an update from the IgA nephropathy classification working group. Kidney Int. 2017;91:1014-1021.

9. Hogg RJ, Lee J, Nardelli N, et al. Clinical trial to evaluate omega-3 fatty acids and alternate day prednisone in patients with IgA nephropathy: Report from the southwest pediatric nephrology study group. Clin J Am Soc Nephrol. 2006;1:467-474.

10. Rauen T, Eitner F, Fitzner C, et al. Intensive Supportive Care plus Immunosuppression in IgA Nephropathy. N Engl J Med. 2015;373:2225-2236.

11. Tumlin JA, Lohavichan V, Hennigar R. Crescentic, proliferative IgA nephropathy: clinical and histological response to methylprednisolone and intravenous cyclophosphamide. Nephrol Dial Transplant. 2003;18:1321-1329.

12. Eastwood JB, Corbishley CM, Grange JM. Tuberculosis and the kidney. J Am Soc Nephrol 2001;12:1307-1314.

13. Wen YK, Chen ML. Crescentic glomerulonephritis associated with miliary tuberculosis. Clin Nephrol. 2009; 71(3):310-313.

14. Fofi C, Cherubini C, Barbera G, Nicoletti MCD, Di Giulio S. Extracapillary IgA nephropathy and pulmonary tuberculosis. Clinical Pulmonary Medicine. 2005;12:305-308.

15. Nasr SH, Vivette DA. IgA-Dominant Postinfectious Glomerulonephritis: A New Twist on an Old Disease. Nephron Clinical practice. 2011;119:18-26.

\section{Correspondence to:}

Marta Marques, MD

Serviço de Medicina Interna,

Centro Hospitalar Póvoa de Varzim - Vila do Conde

Largo da Misericórdia, 4490-421 Póvoa de Varzim

E-mail: martamarkes26@hotmail.com 